\title{
Intravenous lidocaine does not affect the anesthetic depth during rapid sequence induction and intubation as assessed by Bispectral Index monitoring: a randomized double blind study
}

\author{
Chryssoula Staikou, Anteia Paraskeva, Iosifina Karmaniolou, Antonis Vezakis, Athanasia Tsaroucha
}

Aretaieio Hospital, Athens, Greece

Submitted: 4 August 2012

Accepted: 11 October 2012

Arch Med Sci 2013; 9, 4: 713-718

DOI: $10.5114 /$ aoms.2013.36898

Copyright @ 2013 Termedia \& Banach

\begin{abstract}
Introduction: We investigated the impact of intravenous lidocaine on anesthetic depth, as assessed by Bispectral Index score (BIS), and hemodynamic responses to rapid sequence induction/intubation.

Material and methods: Eighty-four surgical patients with risk factors for regurgitation/aspiration were randomized to receive either lidocaine $1.5 \mathrm{mg} / \mathrm{kg}$ or normal saline in a double-blind fashion. Propofol $2 \mathrm{mg} / \mathrm{kg}$, lidocaine or normal saline, followed by rocuronium $1 \mathrm{mg} / \mathrm{kg}$ were administered intravenously and trachea was intubated under cricoid pressure application. The BIS scores were recorded before induction of anesthesia, immediately after, at $30 \mathrm{~s}$ and $1 \mathrm{~min}$ after rocuronium injection and every $30 \mathrm{~s}$ after intubation, for $10 \mathrm{~min}$. Systolic/diastolic blood pressure and heart rate were measured before induction, immediately after and at 1 min following rocuronium administration, and every minute for $10 \mathrm{~min}$ after intubation.

Results: Data from 78 patients were analyzed. Demograpic characteristics did not differ between the study groups. A total of 24 BIS scores were recorded for each patient. No difference was found in BIS values between lidocaine and control groups at any time point $(F=2.936, p=0.91)$. Also no difference was detected in heart rate, systolic and diastolic blood pressure at any time point of the study period between the two groups $(F=0.063, p=0.80, F=0.007, p=0.93$, $F=0.435, p=0.51$ respectively). No episodes of significant bradycardia occurred and none of the patients reported awareness/recall of the procedure.

Conclusions: Lidocaine $1.5 \mathrm{mg} / \mathrm{kg}$ given intravenously during rapid sequence induction does not affect BIS values, or blunt the hemodymanic response to laryngoscopy and intubation.
\end{abstract}

Key words: lidocaine, anesthetic depth, bispectral index, rapid sequence anesthesia.

\section{Introduction}

Rapid sequence induction and intubation (RSII) represents the standard airway management technique for patients at high risk for regurgitation and aspiration. Even though several modifications have been proposed [1], the traditional version of the technique is still preferred by many anesthesiologists. The standard process consists of preoxygenation of the lungs with a tightly fitting face mask, followed by rapid sequential administration of an intravenous hypnotic and a fast-acting neuromuscular agent in predeter-
Corresponding author: Chryssoula Staikou MD Aretaieio Hospital 76 Vass Sophias St 11528 Athens, Greece Phone: +302107286195 E-mail: c_staikou@yahoo.gr 
mined doses, while opioids are omitted [1]. Manual ventilation is avoided, and cricoid pressure may be applied during the procedure in order to further reduce the risk of regurgitation, even though the effectiveness of this maneuver is controversial [1].

Excessive hemodynamic response to laryngoscopy and tracheal intubation due to sympathetic firing is a significant problem encountered with RSII. Lidocaine has been used in clinical practice to blunt the hemodynamic response to airway instrumentation due to its suppressive effects on airway reflexes and its antiarrhythmic properties. Nevertheless, the reports about its efficacy in attenuating the sympathetic response to intubation are contradictory [1-5].

Another problem related to RSII is the possibility of light anesthesia with increased risk of awareness and postoperative recall, since the drugs are given rapidly, and the dose of the induction agent is predetermined and not step-by-step titrated according to individual needs, while opioids may be spared. In this regard, brain monitoring of consciousness level, as the Bispectral Index (BIS), has been used to assess the anesthetic depth provided by different induction agents during RSII [6].

Bispectral Index is a well-established method of monitoring anesthetic depth, and consequently a useful tool in assessing the risk of awareness during induction and maintenance of anesthesia. It gives a single, dimensionless number (0 to 100), after algorithmic analysis of patients' electroencephalographic signals. Generally, higher values denote wakefulness, with the range 40 to 60 being considered indicative of an adequate level of anesthesia.

A possible interaction between local and general anesthetics has attracted the interest of investigators; specifically, lidocaine has been found to produce a minimum alveolar concentration (MAC)sparing effect of 10-28\% [7], and also to reduce propofol requirements during total intravenous anesthesia [8]. The combination of drugs with such interaction could be extremely useful if applied in situations with high risk of awareness, such as RSII. Thus, lidocaine could possibly represent a useful adjuvant in RSII, not only by suppressing airway reflexes, but also by increasing the anesthetic depth as a result of its interaction with anesthetic drugs.

To our knowledge the possible utility of lidocaine in increasing the anesthetic depth, and thus reducing the risk of awareness during RSII, has not been studied before. Our hypothesis was that intravenous (IV) lidocaine in non-toxic doses would be associated with deeper levels of anesthesia, and thus lower BIS values, during RSII.

The aim of the present randomized, double blind study was to assess the impact of IV lidocaine $1.5 \mathrm{mg} / \mathrm{kg}$ on BIS values when administered during RSII.

\section{Material and methods}

The study was approved by the Institutional Review Board of Aretaieio Hospital, University of Athens, Greece. The study is also registered in the ClinicalTrials.gov protocol registration system (NCT01238718). Eighty-four patients, ASA I-II, between 20 and 70 years old, with risk factors for regurgitation/aspiration, who presented for surgery under general anesthesia, were included in the study. All patients gave written informed consent to participate in this prospective randomized double blinded study. History of reflux or diagnosed diaphragmatic hernia, emergency procedures, reported full stomach or conditions associated with delayed gastric emptying (pain, trauma) were considered as risk factors for regurgitation/aspiration requiring RSII. Exclusion criteria were neurological disorders or intake of drugs that could affect BIS values, hypertension or other cardiovascular disease under hypertensive or antiarrhythmic therapy, diabetes mellitus, pregnancy, and morbid obesity (body mass index $(\mathrm{BMI})>40 \mathrm{~kg} / \mathrm{m}^{2}$ ). Patients did not receive any premedication, apart from ranitidine $50 \mathrm{mg}$ and metoclopramide $10 \mathrm{mg}$ IV $30 \mathrm{~min}$ before being transferred to the operating room. Patients were randomly assigned to receive lidocaine (group L, $n=42$ ) or normal saline (control, group $C, n=42$ ) by the use of sealed envelopes describing the group of assignment.

After positioning on the operating table, apart from the routine monitoring (ECG, heart rate, pulse oximeter, blood pressure measurement, DatexOhmeda S/5TM Anaesthesia Monitor, Helsinki, Finland), a BIS ${ }^{\mathrm{TM}}$ sensor was attached to the patient's forehead in conjunction with the BIS ${ }^{\mathrm{TM}}$ XP monitor (Model A-2000, Aspect Medical Systems, Inc., Natick, MA 01760, USA). An 18 G vein catheter was used for fluid replacement (Ringer lactated infusion) and anesthetic drug administration.

According to the group allocation, the patients received either lidocaine $1.5 \mathrm{mg} / \mathrm{kg}$ or normal saline, both prepared in a total volume of $10 \mathrm{ml}$ by an independent investigator. After preoxygenation with $100 \% \mathrm{O}_{2}$ for 3 min via a tightly fitting face mask, propofol®-Lipuro $1 \%$ in a dose of $2 \mathrm{mg} / \mathrm{kg}$ was administered IV in $20 \mathrm{~s}$, immediately followed by lidocaine or normal saline and rocuronium $1 \mathrm{mg} / \mathrm{kg}$. One minute after rocuronium injection, without applying manual ventilation, the trachea was intubated under direct laryngoscopy by an experienced anesthesiologist in less than $30 \mathrm{~s}$, under cricoid pressure applied by the same trained and experienced assistant. Patients with difficult intubation, where more than $30 \mathrm{~s}$ or more than one intubation attempt or additional/special equipment was needed, were excluded from the study measurements. After tracheal intubation, intermittent positive pressure ventilation was applied, adjusted to maintain 
end-tidal $\mathrm{CO}_{2}$ within $35-40 \mathrm{~mm} \mathrm{Hg}$ and sevoflurane was administered at $1 \%$ end-tidal concentration in a nitrous oxide-oxygen mixture $\left(\mathrm{FiO}_{2}: 0.45\right)$. Opioids were spared during the study period, thus from baseline measurement until 10 min after tracheal intubation.

\section{Measurements}

Bispectral Index scores were recorded by an investigator blinded to the patient's allocation group, before induction of anesthesia, immediately after rocuronium injection and also at $30 \mathrm{~s}$ and at 1 min after its administration, just before laryngoscopy. After intubation, BIS was assessed at $30 \mathrm{~s}$ after cuff inflation and thereafter every $30 \mathrm{~s}$ for $10 \mathrm{~min}$. A total of $24 \mathrm{BIS}$ scores were recorded for each patient. Heart rate (HR), systolic (SBP) and diastolic blood pressure (DBP) were measured before induction, immediately following rocuronium injection, at 1 min after rocuronium injection, and every minute for the next 10 min after intubation and cuff inflation. Any complications, such as regurgitation, aspiration, desaturation $\left(\mathrm{SpO}_{2}\right.$ less than $90 \%$ ), bradycardia, awareness and recall of the procedure or other adverse effects were recorded.

The impact of lidocaine pretreatment on BIS values during RSII was the primary outcome measure of the study, with the rest of the variables being secondary outcome measures.

Power analysis showed that in order to detect a size effect of $d=0.8\left(\right.$ where $\left.d=\left(\mu-\mu^{\prime}\right) / \sigma\right)$, which corresponded to a $20 \%$ difference in BIS values 1 min after intubation, a total sample size of 68 patients was necessary to have an $\alpha$ error of 0.05 and a power of $80 \%$ in a two-tailed test.

\section{Statistical analysis}

The statistical package SPSS, v. 19.0 was used for the analysis. Demographic data followed normal distribution and were analyzed with the twosample $t$-test. BIS values, HR, SBP and DBP at different time points were analyzed with ANOVA repeated measures. The level of statistical significance was considered an $\alpha$ value of 0.05 .

\section{Results}

Data from 78 patients (40 in the L group and 38 in the $($ group) were analyzed. Two patients of the $L$ group were excluded from data analysis, one due to a problem with airway management (laryngoscopic view Cormack grade 3 , successful intubation on the second attempt) and one due to BIS monitor malfunction. Also, 4 patients of the $C$ group were excluded, one due to intake of lorazepam one hour preoperatively, one due to a problem with airway management (laryngoscopic view Cormack grade 3 , use of an intubation laryngeal mask), and 2 due to protocol violation (midazolam and fentanyl, respectively, were administered before induction).

Demographic data are presented in Table I; the patients did not differ regarding age, height or body weight ( $p=0.768, p=0.509$ and $p=0.315$ respectively). There was no difference in BIS values between the $L$ and $C$ group at any time point of measurement $(F=2.936, p=0.91)$, as shown in Figure 1. Also no difference was detected in HR, SBP and DBP at any time point of measurement between the control and the treatment group $(F=0.063, p=0.80, F=0.007, p=0.93, F=0.435$, $p=0.51$ respectively), as presented in Table II.

Diaphragmatic contraction/movement was observed in 3 patients of the $L$ group and in 2 patients of the $\mathrm{C}$ group during airway instrumentation. Also, $\mathrm{SpO}_{2}$ dropped down to $90 \%$ after completion of tracheal intubation, without further sequelae, in 1 patient of the $L$ group. Even though anticholinergic premedication was not used, bradycardia needing treatment or any other cardiovascular adverse events did not occur in any patient. Also, no patient reported awareness/recall of the procedure when asked postoperatively.

\section{Discussion}

According to our results, IV lidocaine $1.5 \mathrm{mg} / \mathrm{kg}$ did not significantly affect the BIS values in patients undergoing RSII with propofol and rocuronium, at any time point of the study period, thus from induction of anesthesia until 10 min after tracheal intubation. Our results are in agreement with the main finding of Kim et al., who reported that IV lidocaine $1.5 \mathrm{mg} / \mathrm{kg}$ did not affect the hypnotic response to conventional non-rapid sequence intubation, as assessed with BIS [5]. Nevertheless, these authors found that lidocaine was associated with reduced pre-intubation BIS values [5]. On the other hand, we found a trend towards lower post-intubation BIS values in the lidocaine versus control group, without statistical significance. The different study protocols may explain these findings; Kim et al.

Table I. Age, height and body weight in the lidocaine $(L)$ and control $(C)$ group respectively

\begin{tabular}{|llllll|}
\hline Group & $N$ & M/F & Age [years] & Height [cm] & Weight [kg] \\
\hline L & 40 & $6 / 34$ & $45.57 \pm 13.71$ & $165.57 \pm 7.88$ & $65.55 \pm 12.80$ \\
\hline C & 38 & $4 / 34$ & $44.63 \pm 14.40$ & $163.68 \pm 16.10$ & $70.47 \pm 20.70$ \\
\hline
\end{tabular}

$N$-number of patients analyzed, M/F-male/female. Data are mean \pm standard deviation 


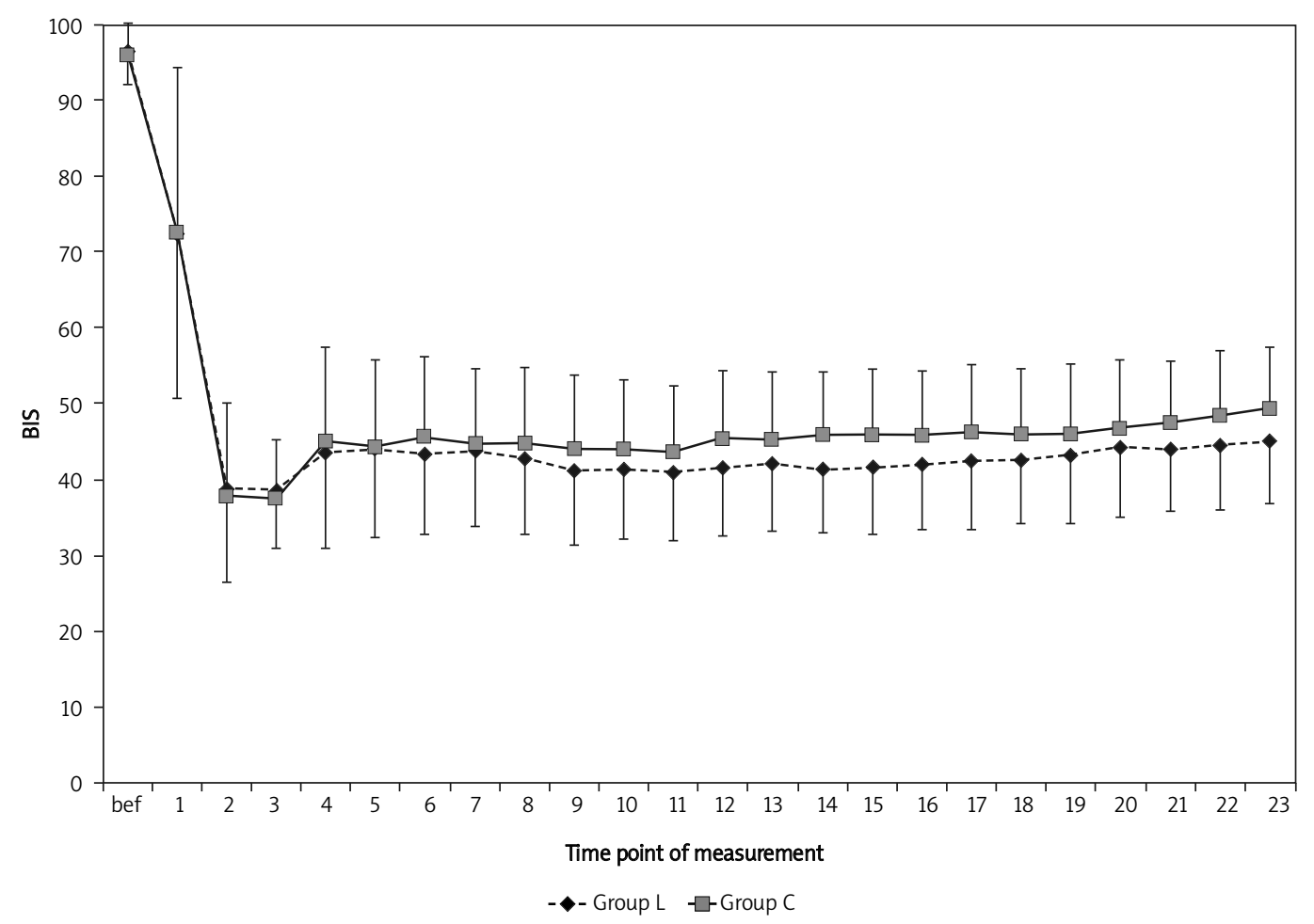

Figure 1. Bispectral Index values at the time points of measurement, in lidocaine (L) and control (C) groups. Values are mean \pm SD

Time points of measurements: bef: before induction of anesthesia, 1-immediately after rocuronium, 2-30 s after rocuronium, 3-1 min after rocuronium - just before laryngoscopy, 4-30 s after cuff inflation, 5-22 - every 30 s, 23-10 min after cuff inflation

Table II. Systolic (SAP), diastolic blood pressure (DAP) and heart rate (HR) during the time points of measurement in the lidocaine (L) and control (C) group respectively

\begin{tabular}{|c|c|c|c|c|c|c|c|c|c|c|c|c|c|}
\hline $\begin{array}{l}\text { Time } \\
\text { points }\end{array}$ & Bef & 1 & 2 & 3 & 4 & 5 & 6 & 7 & 8 & 9 & 10 & 11 & 12 \\
\hline \multicolumn{14}{|l|}{ SAP } \\
\hline $\mathrm{L}$ & $130 \pm 18$ & $118 \pm 17$ & $108 \pm 13$ & $138 \pm 21$ & $128 \pm 19$ & $120 \pm 18$ & $115 \pm 16$ & $111 \pm 14$ & $108 \pm 12$ & $105 \pm 11$ & $104 \pm 12$ & $103 \pm 13$ & $103 \pm 11$ \\
\hline C & $112 \pm 14$ & $146 \pm 25$ & $132 \pm 21$ & $121 \pm 18$ & $114 \pm 18$ & $112 \pm 14$ & $110 \pm 14$ & $107 \pm 14$ & $106 \pm 13$ & $105 \pm 12$ & $106 \pm 12$ & $103 \pm 13$ & $103 \pm 12$ \\
\hline \multicolumn{14}{|l|}{ DAP } \\
\hline L & $74 \pm 11$ & $70 \pm 12$ & $67 \pm 11$ & $89 \pm 17$ & $79 \pm 13$ & $72 \pm 14$ & $68 \pm 14$ & $65 \pm 11$ & $63 \pm 12$ & $61 \pm 12$ & $59 \pm 13$ & $61 \pm 13$ & $60 \pm 13$ \\
\hline C & $73 \pm 11$ & $72 \pm 11$ & $67 \pm 9$ & $92 \pm 15$ & $81 \pm 15$ & $73 \pm 13$ & $68 \pm 13$ & $63 \pm 12$ & $65 \pm 12$ & $63 \pm 11$ & $62 \pm 11$ & $61 \pm 11$ & $61 \pm 11$ \\
\hline \multicolumn{14}{|l|}{$\mathrm{HR}$} \\
\hline L & $85 \pm 14$ & $76 \pm 13$ & $94 \pm 14$ & $91 \pm 13$ & $89 \pm 13$ & $89 \pm 13$ & $87 \pm 12$ & $85 \pm 12$ & $82 \pm 12$ & $81 \pm 12$ & $79 \pm 11$ & $78 \pm 11$ & $77 \pm 11$ \\
\hline$C$ & $81 \pm 15$ & $78 \pm 17$ & $96 \pm 16$ & $92 \pm 13$ & $92 \pm 13$ & $89 \pm 13$ & $86 \pm 13$ & $85 \pm 13$ & $83 \pm 13$ & $80 \pm 12$ & $77 \pm 12$ & $75 \pm 13$ & $74 \pm 13$ \\
\hline
\end{tabular}

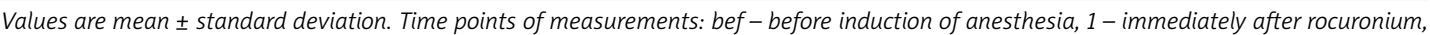
2-1 min after rocuronium-just before laryngoscopy, 3-1 min after cuff inflation, 4-11 - every 1 min, 12 - 10 min after cuff inflation

used thiopental as an induction agent with relatively high BIS scores and performed laryngoscopy 2 min after rocuronium injection [5], while we applied an RSII anesthetic protocol with propofol/ rocuronium. It has been demonstrated that in RSII propofol is associated with deeper levels of anesthesia compared to thiopental [6]; in our study preintubation BIS values were quite low (about 40) in both groups and possible small differences due to lidocaine were not identifiable.
Our findings are in accordance with those of Nakayama et al., who reported that premixing lidocaine with propofol reduces injection pain without affecting the BIS responses [9]. Also, Gottschalk et al. reported that IV lidocaine decreases BIS only in the presence of midazolam, suggesting that the effect of lidocaine on BIS results from modulation by midazolam and not by a direct effect exerted by lidocaine [10]. In our study opioids and other depressants were omitted in order to minimize the effects 
of other drugs on BIS values and have a more clear picture about the effects of lidocaine on BIS. Also, even though modifications of the RSII procedure have been proposed and advocated in clinical practice, opioids are omitted in the classic RSII procedure [1].

Systemic lidocaine has probably a significant depressant effect on the central nervous system (CNS) [11]. This central action of lidocaine can possibly explain the reported decrease of BIS values down to 0 for $15 \mathrm{~min}$ after an inadvertent lidocaine overdose [12]. Probably, high dosing, synergism with other central sedatives, such as benzodiazepines or opioids, and optimal timing of administration can maximize the depressant efficacy of lidocaine. Regarding timing, in our study lidocaine was administered about 1.5 min before intubation. Also, usually in clinical practice and in most studies, lidocaine is administered 1 to $2 \mathrm{~min}$ before airway instrumentation [3, 5]. Nevertheless, it has been suggested that at least three or more minutes are probably required for lidocaine to achieve its maximal efficacy, in terms of suppression of airway reflexes $[1,13]$.

Regarding the secondary outcomes of our study, no difference was detected in HR, SBP and DBP, between $L$ and $C$ groups, at any time point. Hypertension and tachycardia following airway instrumentation are more pronounced during RSII due to excessive catecholamine release. We found that lidocaine was ineffective in blunting RSII hemodynamic responses. Our results are in agreement with those of previous studies reporting that IV lidocaine $1.5-2 \mathrm{mg} / \mathrm{kg}$ does not attenuate the tachycardia and hypertension associated with rapid sequence induction and intubation $[2,3,14]$. In our study, patients did not receive any anticholinergic premedication, in order to avoid any influence on the results. We observed no significant bradycardia and/or hypotension during the pre-intubation period, thus following anesthetic induction, even in the propofol/lidocaine group. The most likely explanation is the use of rocuronium, which has mild vagolytic effects and may attenuate BP decreases via increasing the HR and thus cardiac output [15]. In our study, a HR increase was evident in both groups following rocuronium administration. Also, the relatively small dose of lidocaine and the short time interval between anesthetic induction and laryngoscopy may have played a role.

The sympathetic firing due to laryngoscopy/intubation and depth of anesthesia/unconsciousness as assessed by BIS are not strictly related. BIS values reflect cerebral cortical activity [16], while the neural reflexes associated with the hemodynamic responses to laryngoscopy and intubation occur predominantly at the subcortical level, specifically in brain stem and hypothalamus, and thus they may be unrelated to the BIS values [17]. Even though BIS values are associated with the anesthetic depth and risk of awareness, they may not be of special value in predicting the hemodynamic responses to airway instrumentation.

The displayed BIS values lag the patient's electroencephalographic state by about 10-15 s [18]. Nevertheless, we consider that this delay has not affected our results, since it existed in measurements of both groups, while BIS recordings were made in short intervals (every $30 \mathrm{~s}$ ) in order to have an adequate number of BIS scores, thus 24 BIS values for each patient, for comparisons; no differences were found between the groups at any time point.

A lidocaine dose of $1.5 \mathrm{mg} / \mathrm{kg}$ was preferred in our study, since it has been used in several trials as a preintubation adjuvant for rapid and conventional non-rapid sequence induction [3-5, 13, 14]. The rocuronium dose we used was $1 \mathrm{mg} / \mathrm{kg}$, since this or higher doses are indicated in RSII in order to provide satisfactory intubation conditions in $1 \mathrm{~min}$ [19, 20]. Cricoid pressure was applied in all cases by the same trained and experienced assistant, in order to ensure that the technique is performed correctly and to minimize possible differences in the exerted force among the patients.

The results of our study are possibly affected by the timing of lidocaine administration relatively to intubation, which was 1.5 min before. Since we investigated the efficacy of lidocaine in RSII, the study protocol and timing were analogous to those used in emergency situations and comparable previous studies, thus 1-2 min before laryngoscopy. Also, the study was designed to be double blind, so we had to administer lidocaine after propofol in order to avoid propofol injection pain in the control group, which could reveal group allocation. Propofol®-Lipuro (B. Braun Melsungen AG, Germany) was preferred for ethical reasons, since it has been associated with reduced pain intensity at injection [21].

In conclusion, under the present study design we found that lidocaine $1.5 \mathrm{mg} / \mathrm{kg}$ does not affect the pre- or post-intubation BIS values during RSII with propofol and rocuronium. We also found that lidocaine was ineffective in blunting the hemodynamic response to rapid sequence intubation. According to our findings, lidocaine does not offer any clinical advantage over placebo, in terms of anesthetic depth and hemodynamic stability, when used as an adjuvant in rapid sequence induction and intubation.

\section{References}

1. El-Orbany M, Connolly LA. Rapid sequence induction and intubation: current controversy. Anesth Analg 2010; 110: 1318-25.

2. Pathak D, Slater RM, Ping SS, From RP. Effects of alfentanil and lidocaine on the hemodynamic responses to 
laryngoscopy and tracheal intubation. J Clin Anesth 1990; 2: 81-5.

3. Chraemmer-Jørgensen B, Høilund-Carlsen PF, Marving J, Christensen V. Lack of effect of intravenous lidocaine on hemodynamic responses to rapid sequence induction of general anesthesia: a double-blind controlled clinical trial. Anesth Analg 1986; 65: 1037-41.

4. Yorukoglu D, Asik Y, Ökten F. Rocuronium combined with i.v. lidocaine for rapid tracheal intubation. Acta Anasthesiol Scand 2003; 47: 583-7.

5. Kim WY, Lee YS, Ok SJ, et al. Lidocaine does not prevent bispectral index increases in response to endotracheal intubation. Anesth Analg 2006; 102: 156-9.

6. Sie MY, Goh PK, Chan L, Ong SY. Bispectral index during modified rapid sequence induction using thiopentone or propofol and rocuronium. Anaesth Intensive Care 2004; 32: $28-30$.

7. Himes RS, DiFazio CA, Burney RG. Effects of lidocaine on the anesthetic requirements for nitrous oxide and halothane. Anesthesiology 1977; 47: 437-40.

8. Altermatt FR, Bugedo DA, Delfino AE, et al. Evaluation of the effect of intravenous lidocaine on propofol requirements during total intravenous anaesthesia as measured by bispectral index. Br J Anaesth 2012; 108: 979-83.

9. Nakayama M, Ichinose H, Yamamoto $S$, et al. The effect of lidocaine on the bispectral index during anesthesia induction with propofol. Masui 2001; 50: 1213-6.

10. Gottschalk A, McKay AM, Malik ZM, Forbes M, Durieux ME, Groves DS. Systemic lidocaine decreases the Bispectral Index in the presence of midazolam, but not its absence. J Clin Anesth 2012; 24: 121-56.

11. Nishino T, Hiraga K, Sugimori K. Effects of IV lignocaine on airway reflexes elicited by irritation of the tracheal mucosa in humans anaesthetized with enflurane. $\mathrm{Br} J$ Anaesth 1990; 64: 682-7.

12. Gaughen CM, Durieux M. The effect of too much intravenous lidocaine on bispectral index. Anesth Analg 2006; 103: 464-5.

13. Lev R, Rosen P. Prophylactic lidocaine use preintubation: a review. J Emerg Med 1994; 12: 499-506.

14. Kim JT, Shim JK, Kim SH, et al. Remifentanil vs. lignocaine for attenuating the haemodynamic response during rapid sequence induction using propofol: double-blind randomised clinical trial. Anaesth Intensive Care 2007; 35: 20-3.

15. Stevens JB, Hecker RB, Talbot JC, Walker SC. The haemodynamic effects of rocuronium and vecuronium are different under balanced anaesthesia. Acta Anaesthesiol Scand 1997; 41: 502-5.

16. Liu J, Singh H, White PF. Electroencephalographic bispectral index correlates with intraoperative and depth of propofolinduced sedation. Anesth Analg 1997; 81: 185-9.

17. Mi WD, Sakai T, Takahashi S, Matsuki A. Haemodynamic and electroencephalograph responses to intubation during induction with propofol or propofol/fentanyl. Can J Anaesth 1998; 45: 19-22.

18. Pilge S, Zanner R, Schneider G, Blum J, Kreuzer M, Kochs EF. Time delay of index calculation: analysis of cerebral state, bispectral, and narcotrend indices. Anesthesiology 2006; 10: 488-94.

19. Andrews J, Kumar N, van den Brom RH, Olkkola KT, Roest GJ, Wright PM. A large simple randomized trial of rocuronium versus succinylcholine in rapid-sequence induction of anaesthesia along with propofol. Acta Anaesthesiol Scand 1999; 43: 4-8.

20. Perry JJ, Lee JS, Sillberg VA, Wells GA. Rocuronium versus succinylcholine for rapid sequence induction intubation. Cochrane Database Syst Rev 2008; CD002788.
21. Sun NC, Wong AY, Irwin MG. A comparison of pain on intravenous injection between two preparations of propofol. Anesth Analg 2005; 101: 675-8. 